\title{
Dynamic CDBR for Underwater Wireless Sensor Networks
}

\author{
Parul Garg \\ Department of Computer Science \& Engineering \\ Guru Nanak Dev University \\ Amritsar
}

\author{
Sandeep Waraich \\ Department of Computer Science \& Engineering \\ Guru Nanak Dev University \\ Amritsar
}

\begin{abstract}
The UWSN has found their place in many applications like sea exploration, flood detection and many more. The sensor nodes used in UWSN are installed with specialized depth sensors to compute the depth value of itself with respect to sink that is deployed at the surface. Obviously, to transfer data by sensor nodes to sink, some routing protocol is necessary for communication. There are many routing protocols like DBR, CDBR, VBF, ICRP, DDD, H2H-DAB and many more. Some of them need location information while some need depth data to make routing decision to forward data. Since CDBR protocol is implemented for static network topology, in this paper, CDBR for dynamic network topology means the positions of the sinks keeps on changing with successive rounds is implemented. The simulations are done in MATLAB and simulation graphs are drawn for network lifetime, packets transferred, end to end delay and remaining energy. The graphs show that the proposed algorithm outperforms the existing algorithm.
\end{abstract}

\section{Keywords}

Acoustic nodes, data forwarding, depth based routing, multiple sink architecture, UWSN.

\section{INTRODUCTION}

The underwater wireless sensor networks incorporate the usage of number of sensor nodes and base stations that are installed under sea for many applications like sea exploration, flood detection and many more. These sensor nodes communicate through acoustic signals due to high propagation delay of radio signals. These sensor nodes send the data to the base station (deployed at surface of sea) and from that base station, user retrieves the information. The characteristics of underwater enforces distinctive necessities on algorithms and protocols intended for underwater wireless sensor networks [10]. These sensor nodes make use of many routing algorithms to transfer data from one node to other. The information needed by routing protocols include the location information of the sensor nodes and some need the depth information. Depth threshold delimits the number of qualified neighbors for data forwarding by picking neighbors in a particular range [11]. For calculating the depth information, sensor nodes are equipped with depth sensors. There are many routing protocols that are used by sensor nodes like depth based routing protocol, vector based forwarding, information carrying routing protocol, dynamic address based routing protocol and constraint based depth based routing protocol. The figure 1 shows the general scenario of underwater wireless sensor networks in which number of sensor nodes are deployed at the bottom of sea and number of acoustic nodes are deployed at different depths and sinks are anchored at the surface of sea.

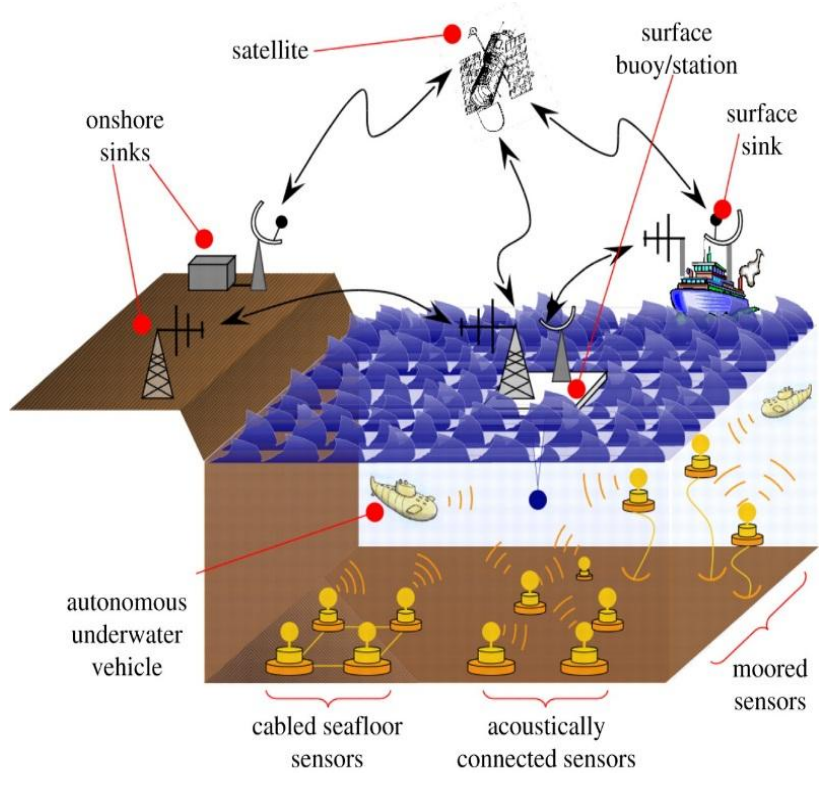

Figure 1. General scenario of underwater wireless sensor network [1].

\section{RELATED WORK}

In [1], Q-Learning based tracking technique was used to find the next forwarding node on the basis of residual energy of the individual node in order to reduce the number of forwarding nodes and energy consumption and in addition the buffer size is also considered for finding the next forwarder to reduce the packet drop. In [2], the author has proposed a depth-based routing (DBR) protocol. DBR makes use of depth information of sensor nodes. In [3], the authors have proposed various DTN routing techniques for different types of DTN routing schemes in UWSNs and surveyed state-of-the-art DTN routing protocols, and analyzed the detailed information in order to draw up a comparison table. In [4], the authors have proposed depth based multi hop routing protocol. In [5], the authors have implemented protocol called Advance Energy Efficient Depth Based Routing protocol (AEEDBR) which not only distributes energy evenly throughout the network for all the nodes but also helps to improve network lifetime. In [6], the authors proposed an energy efficient routing protocol, named (energy-efficient depth-based routing protocol) EEDBR for UWSNs. EEDBR utilizes the depth of sensor nodes for forwarding data packets and the residual energy of sensor nodes is also taken into account to improve the network lifetime. In [7], authors have proposed an extension of depth based routing protocol called depth based multi hop routing protocol. In [8], authors have proposed Delay-Sensitive Depth-Based Routing (DSDBR), DelaySensitive Energy Efficient Depth-Based Routing (DSEEDBR) 
and Delay-Sensitive Adaptive Mobility of Courier nodes in Threshold-optimized Depth-based routing (DSAMCTD) protocols. In [9], authors have extended the DBR protocol by limiting the number of forwarding nodes and have extended the network lifetime and energy consumption of the DBR. In [10], the authors have identified the confinements of various routing algorithms used for UWSNs and the design issues for efficient routing algorithm were also discussed. In [11], the authors have analyzed two major acoustic propagation models of Thorp and Monterey-Miami Parabolic Equation (MMPE) to determine the packet drop for DBR, EEDBR, AMCTD, and IAMCTD.

\section{ROUTING PROTOCOLS FOR UWSNs}

There are many routing protocols that are used in transmitting the information among nodes in underwater wireless sensor networks. Some of them are discussed as below:

\subsection{Depth Based Routing Protocol}

DBR is a keen algorithm that tries to transmit a data packet from a source node to multiple sinks [4]. By the word multiple sinks mean, the DBR protocol encompasses the use of multiple sink architecture in which a predetermined number of sinks are to be anchored at the surface of sea. DBR only uses the depth information of nodes. To attain the depth of current node, each sensor node is furnished with a depth sensor. DBR makes a decision for routing by considering depth data, and progresses the data packets from higher depth nodes to lower depth nodes. There is a lack of depth information of immediate nodes and their residual energy, as a result DBR have the possibility of redundant transmissions [6]. When a node has some data to be sent, it will first sense its present depth position with respect to the surface and compress it in the packet header and broadcast it. The receiving node will accelerate this packet by first calculating its depth position and if its depth is smaller than the value compressed in the packet, it will basically avoid the packet. Packets reached at any of the multiple sinks are considered as successful transfer of data and at the last these multiple destination and information sinks can correspond productively through radio channel. The primary points of interest of DBR are as per the following. 1) It doesn't incorporate the use of location data. 2) It deals with dynamic network systems with great energy productivity. 3) It uses multiple sink system without any additional expense [2]. It has a few confinements. Initially, DBR fails to achieve high transmission proportions in sparse territories. Second, broadcasting leads to diminish the performance of the whole system. Third, number of repetitive information transmission are done due to flooding mode [7].

\subsection{Constraint Based Depth Based Routing Protocol}

Most of the routing schemes make use of location data of the sensor nodes in the system, which is a big challenge for UWSNs. On the other hand, CDBR only needs the depth information of sensor nodes. Since depth information is in a two-dimensional space and its computation does not require more transmission power, transmission time and complex mathematical formulas [3]. In DBR protocol, the large propagation delay is introduced because of the occurrence of long distance transmissions between the sensor nodes

\subsection{CDBR FOR DYNAMIC NETWORK TOPOLOGY}

The proposed algorithm also does not incorporate the use of location information. It only needs the depth information of especially in the medium-depth region [8]. The major confinements with the DBR protocol are:

- High propagation delay.

- High energy consumption.

- Redundant data transmission.

These problems are resolved by the authors of [9]. They proposed an efficient routing protocol named constraint based depth based routing protocol (CDBR). The sensor nodes are positioned randomly under the water. They have ignored the horizontal mobility and made an assumption about the depth of the nodes that it does not change till the end of the network exists. A predetermined number of sinks are installed on the water surface with fixed positions and it is the responsibility of nodes to sense data and then deliver the data to sinks when asked for transmission by the forwarding nodes. The RF modems and acoustic modems are installed within the multiple sinks so that they can be able to communicate with the sensor nodes and with each other. The sinks do communication among themselves using the RF Modems. Data received by any of the sinks is considered to be the successful delivery of data. In CDBR, each node keeps a priority Queue P and a data history cradle (DHC). DHC records the information of newest data packets transmitted by a node [5]. The protocol works in two phases:

- Optimal forwarder node set selection

- Forwarding node selection

\subsubsection{Optimal forwarder node set selection:}

The first phase starts with the identification of each node's neighbors. The nodes for which the depth value is less than the depth of current node are said to be its neighbors. There is a limit imposed on number of neighboring nodes that is constrained by a global parameter named depth threshold. The nodes having high value of depth than depth threshold will be allowed to get the data. Depth threshold can be computed as the subtraction of depth of source and neighboring node. A set of nodes known as an optimal forwarder node set is identified by the current node. It should be kept in mind to check whether the current node is in the range of any sink or not. If any of the multiple sinks is in its communication range, the node directly delivers the data to sink, otherwise, it will forward it to its next hop forwarder node set. At the end, one node from the node set is chosen to broadcast data to the next hop forwarder node set.

\subsubsection{Forwarding node selection}

The current node first chooses a set of nodes that are in its communication range and this recognized set is called as optimal forwarder node set. It is to be noted that this set consists of only those sensor nodes that received the sensed information broadcasted by the current node. To do the main task of data forwarding, CDBR chooses a node from this set. The chosen node have the property that its depth value is minimum among the depth values of the other nodes present in the optimal forwarder node set.

the sensor nodes. The sensor nodes are equipped with depth sensors to calculate the depth information. Since the existing CDBR routing does not consider the dynamic network topology, so the proposed algorithm is implemented for dynamic network topology. This proposed algorithm also uses 
the multiple sink architecture. The multiple sinks are anchored randomly and the forwarding nodes are also deployed randomly. The algorithm works like: first a sink activates a forwarding node and then the forwarding node will broadcast the message to the sensor nodes, if any node wants to send data. If any node has sensed some data, it forwards the data to the forwarding node and then the forwarding node will send the aggregated data to the sink. The activation of the forwarding node through the sink is done randomly. The following section describes the methodology of the proposed algorithm.

\section{METHODOLOGY}

Phase 1. Since the proposed routing protocol uses the multiple sink architecture so the very first phase is to deploy the number of sinks randomly. The multiple sink architecture uses number of sinks that are to be deployed at the surface of the sea and number of sensor nodes are to be deployed at different depths in the sea. The below figure shows the general scenario of the multiple sink architecture:

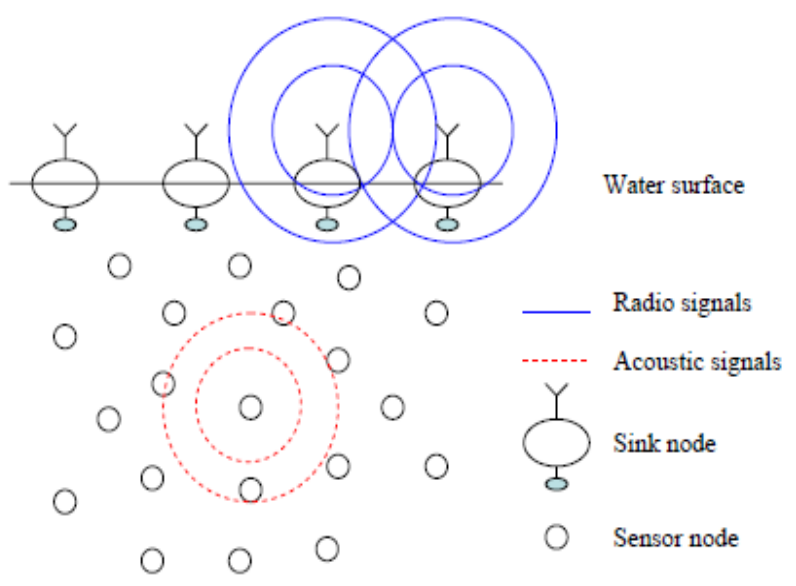

Figure 2. Multiple sink architecture (source [2]).

Phase 2. The proposed protocol uses the concept of forwarding nodes to send data to the sinks. The forwarding nodes are simply the sensor nodes but are chosen as forwarding nodes based on the depth threshold value. The depth threshold value is any default value chosen for the network. The forwarding node is chosen as discussed below: first, the sensor node recognize its neighbors and the nodes having depth less than the current sensor node depth value will be the forwarding nodes. Among these set of nodes, an optimal forwarder is chosen according to the depth threshold value. It is the responsibility of forwarding node to deliver data to any of the sink which is nearer to its position. The placement of the forwarding nodes is also done randomly.

Phase 3. The sensor network consists of number of sensor nodes that are capable of sensing, performing computation. The depth sensors are installed in sensor nodes to calculate the depth value of other sensor nodes and to make a comparison of its value with its own depth so that the routing decision can be made. After the deployment of sinks and forwarding nodes, sensor nodes sense data from the environment and send the data to the acoustic nodes.

Phase 4. The sensed data is collected by the acoustic nodes that send data to the forwarding nodes. Acoustic nodes are also known as the courier nodes which keeps on moving with the water currents and collect data from the nearby sensor nodes. Courier nodes manage their vertical and horizontal movement to collect data from the other nodes. Acoustic nodes and the sensor nodes communicate through acoustic signals and send data to one of the forwarding nodes.

Phase 5. The forwarding nodes perform data aggregation techniques on the collected data from various sensor nodes so that further sending process can be performed. The forwarding node send the aggregated data from the acoustic nodes to the sink to which it is nearer. The communication is done using acoustic signals because radio signals do not work underwater due to high propagation delays.

Phase 6. The evaluation of energy dissipation is done and remaining energy is calculated. Remaining energy is defined as the energy left after all the nodes send data to the destination. The energy is calculated in joules.

Phase 7. If the remaining energy of the sensor node is less than or equal to zero, it will start to count the node as dead otherwise it will return to phase 2 and start the process again. If the dead count is same as the number of nodes taken initially then network lifetime is returned otherwise, it will return to the phase 2 and whole of the process is repeated till the last node dies.

Network Setup

The initial setup of the network is described in the table as shown below:

\begin{tabular}{|l|l|}
\hline Parameter & Initial value \\
\hline Initial energy & 0.01 \\
\hline Network area & 150 X 150 \\
\hline Number of sinks & 4 \\
\hline Number of forwarding nodes & 5 \\
\hline Number of rounds & 1000 \\
\hline Transmitter energy & $50 * 0.000000001$ joules \\
\hline Receiver energy & \\
\hline $\begin{array}{l}\text { Amplification energy when d } \\
\text { is less than d0 }\end{array}$ & $10 * 0.0000000000001$ \\
\hline
\end{tabular}

Initially, 20 number of nodes are taken and each node has initial energy of 0.01 joules. The sensor nodes are deployed randomly. There are 4 sinks that are kept fixed in the existing algorithm and are randomly deployed in the proposed algorithm. The forwarding nodes are shown in pink color and are 5 in number and are kept fixed in existing algorithm and are randomly deployed in the proposed algorithm. The simulations are done by varying the number of nodes. The maximum number of nodes taken are 200. Below figure shows the network setup for existing algorithm in which black circles show the sensor nodes and pink circles shows the 
forwarding nodes and star shows the sink. Black colored small circles show the acoustic nodes.

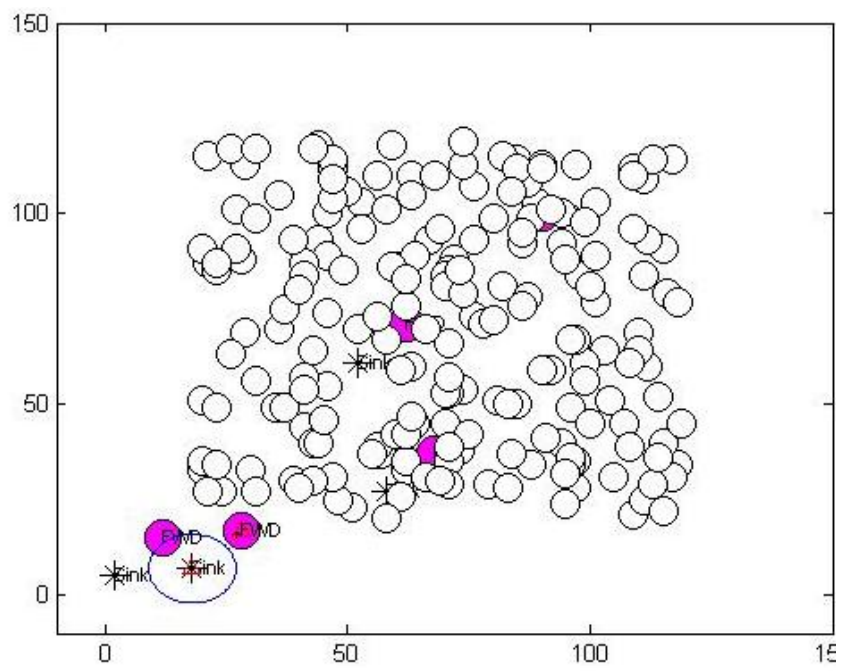

Figure 4. Network setup for proposed algorithm.

\section{RESULTS \& DISCUSSION}

The simulations are done in MATLAB. The parameters onto which results are built are discussed as below:

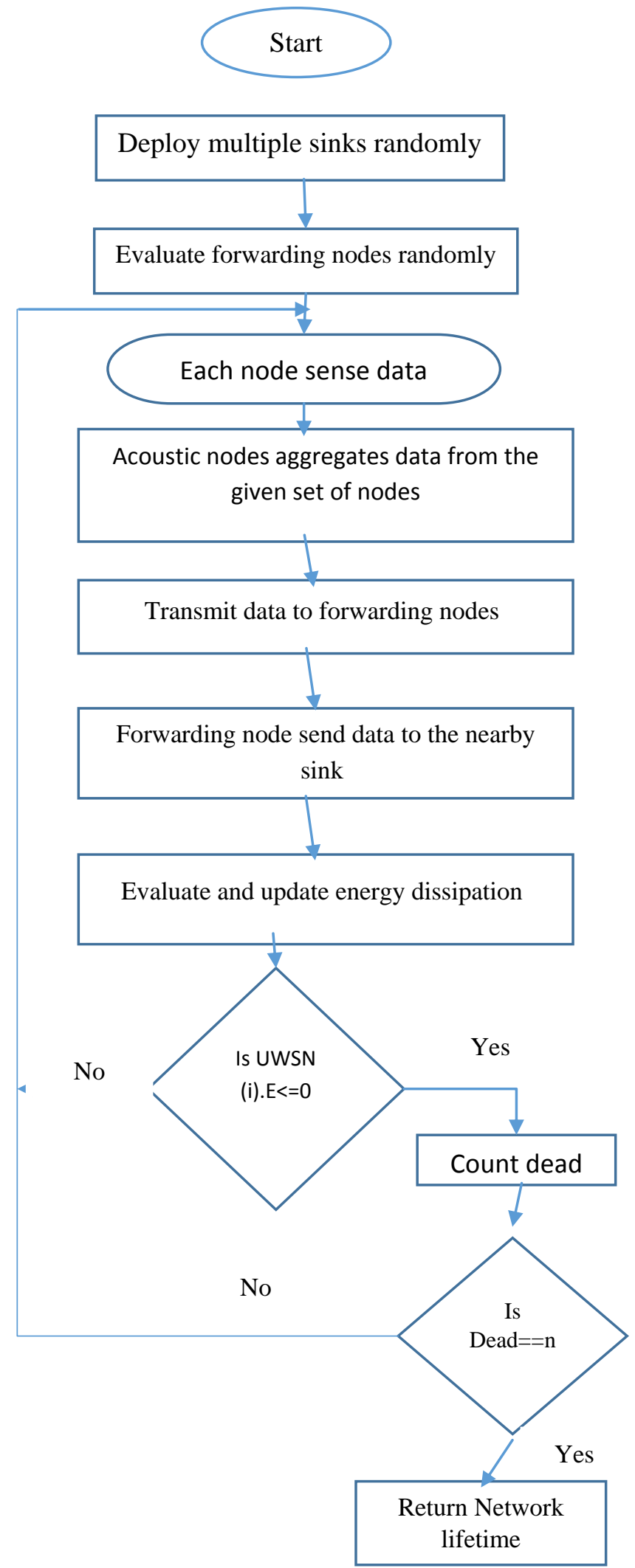

Network Lifetime: It is the amount of time for which an UWSN would be fully functioning. It can also be defined as the time at which the first network node runs out of energy to transmit a packet. Another way to define it comprises the time in which some nodes could die whenever other network nodes 
could be used to gather desired information or to forward information to their destination. The simulation graph is shown as below:

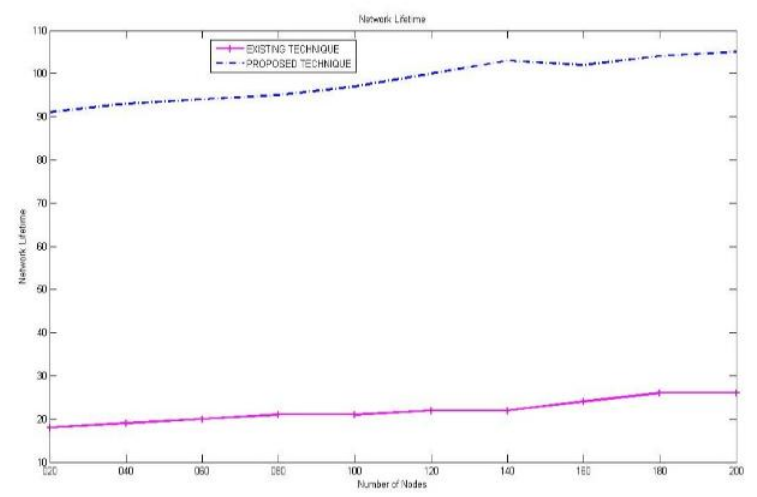

Figure 5. Graph showing network lifetime for existing and proposed algorithm.

The above graph shows that the network lifetime for existing algorithm is very less than the network lifetime for proposed algorithm. It is clear from the graph that the maximum network lifetime for existing algorithm is 26 rounds whereas for proposed algorithm it is 105 rounds.

Packets transferred: It is defined as the number of the packets that are transferred during the simulations. In our results, packets transferred is the sum total of all the packets sent to all sinks. The below graph shows the number of data packets transferred in existing algorithm and proposed algorithm. It is clear that maximum number of packets transferred for existing algorithm is 694477 whereas for proposed algorithm, it is 1633533 which is much greater than the existing one. This shows that proposed algorithm outperforms the existing algorithm. The simulation results are shown below in the line graph:

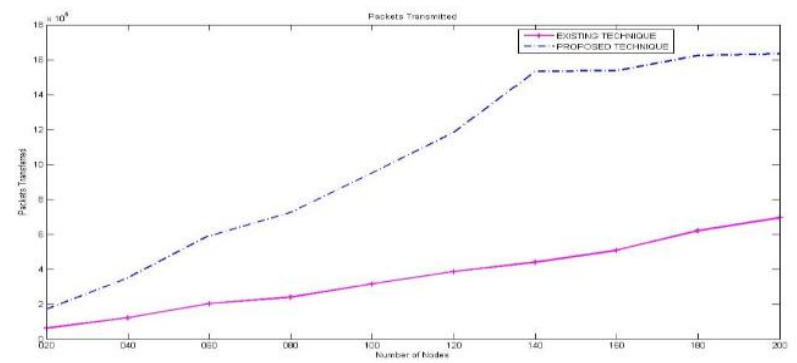

Figure 6. Graph showing packets transferred for existing and proposed algorithm.

End- to- end delay: It is defined as the average time taken by a data packet to reach to the destination. It also incorporates the delay created due to path detection process and the queue in data packet communication. Only the data packets that successfully delivered to destinations that counted. The simulation results are shown below in the line graph:

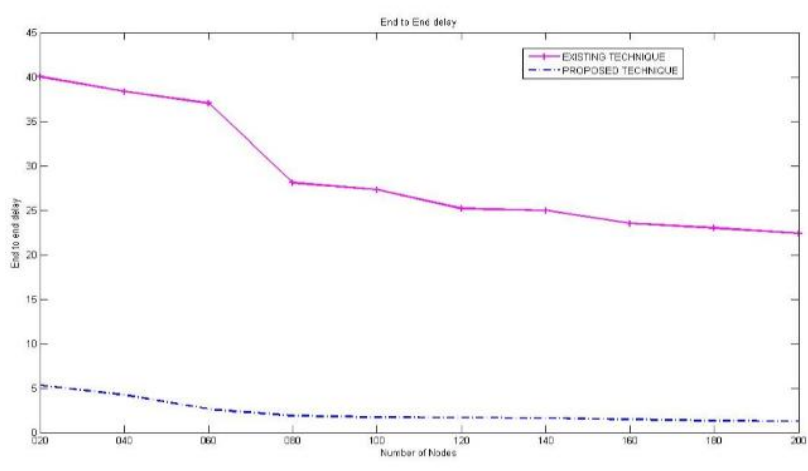

Figure 7. Graph showing end to end delay for existing and proposed algorithm.

The above graph shows the end to end delay for existing and proposed algorithm. It is clear that end to end delay for existing algorithm is 22.4019 whereas for proposed algorithm, it is 1.2899 . This shows that proposed algorithm outperforms the existing algorithm.

Remaining energy: It is defined as the energy left after all the nodes send data to the destination. The remaining energy is calculated in joules. The above graph shows the remaining energy for existing and proposed algorithms. The maximum energy for existing algorithm is 0.0033 whereas for proposed algorithm, it is 0.0097 . This shows that proposed algorithm outperforms the existing algorithm. The simulation results are shown below in the line graph:

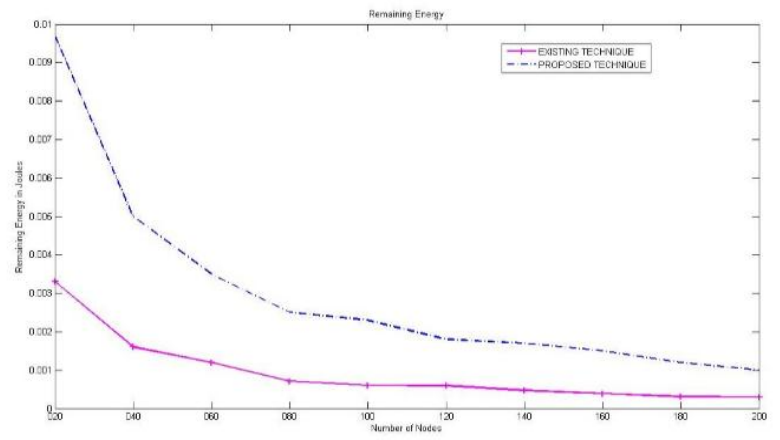

Figure 8. Graph showing remaining energy for existing and proposed algorithm.

\section{CONCLUSIONS}

The network of underwater wireless sensor network consists of variable number of sensor nodes installed at different depths. The sinks are deployed at the water surface and sensor nodes send data to sinks with the help of appropriate routing protocol. The depth based routing protocol uses the multiple sink architecture. Data delivered to any of the sink is considered to be successful. CDBR uses the same approach of DBR but the only restriction is applied on number of the forwarding nodes that is constrained with the concept of depth threshold. The existing network of CDBR considered the sinks located at sea surface with fixed position that is not realistic in nature and in addition, it does not give the information about the number of multiple sinks. The existing network considered the static network topology. This paper has implemented CDBR protocol for multiple sinks with varying locations and evaluate its performance. The simulations results clearly show that the proposed technique outperforms over the available techniques. In near future we 
will modify the proposed technique using the swarm based techniques to enhance the depth based routing technique. Also the use of fuzzy based techniques will also be done to improve the decision making of acoustic nodes.

\section{REFERENCES}

[1] Thangarajan, R., T. Siva, and R. Boopalachakaravarthy. "Adaptive Energy Efficient Routing Protocol with Extended Lifetime in Underwater Sensor Networks." In Communication Systems and Network Technologies (CSNT), 2013 International Conference on, pp. 322-326. IEEE, 2013.

[2] Yan, Hai, Zhijie Jerry Shi, and Jun-Hong Cui. "DBR: depth-based routing for underwater sensor networks." In NETWORKING 2008 Ad Hoc and Sensor Networks, Wireless Networks, Next Generation Internet, pp. 72-86. Springer Berlin Heidelberg, 2008.

[3] Cho, Hsin-Hung, Chi-Yuan Chen, Timothy K. Shih, and Han-Chieh Chao. "Survey on underwater delay/disruption tolerant wireless sensor network routing." IET Wireless Sensor Systems 4, no. 3 (2014): 112-121.

[4] Mahapatro, Gurucharan, and U. Devee Prasan. "Energy Efficient Multiple Sink Variation To The Depth-Based Route Protocol For Under Water Sensor Network." (2012).

[5] Najeeb, F., Z. A. Khan, U. Qasim, Z. Najam, J. Ikram, S. H. Ahmed, M. J. Ashraf, and N. Javaid. "A New Advanced Energy Efficient Routing Protocol for UWSNs." (2014).
[6] Wahid, Abdul, and Dongkyun Kim. "An energy efficient localization-free routing protocol for underwater wireless sensor networks." International journal of distributed sensor networks 2012 (2012).

[7] Guangzhong, Liu, and Li Zhibin. "Depth-based multihop routing protocol for underwater sensor network." In Industrial Mechatronics and Automation (ICIMA), 2010 2nd International Conference on, vol. 2, pp. 268-270. IEEE, 2010.

[8] Jafri, Mohsin Raza, Muhammad Moid Sandhu, Kamran Latif, Zahoor Ali Khan, Ansar Ul Haque Yasar, and Nadeem Javaid. "Towards delay-sensitive routing in underwater wireless sensor networks." Procedia Computer Science 37 (2014): 228-235.

[9] Mahmood, S., H. Nasir, S. Tariq, H. Ashraf, M. Pervaiz, Z. A. Khan, and N. Javaid. "Forwarding Nodes Constraint based DBR (CDBR) and EEDBR (CEEDBR) in Underwater WSNs." Procedia Computer Science 34 (2014): 228-235.

[10] Bayrakdar, Yonca, Nirvana Meratnia, and Aylin Kantarci. "A comparative view of routing protocols for underwater wireless sensor networks." In OCEANS, 2011 IEEE-Spain, pp. 1-5. IEEE, 2011.

[11] Jafri, M. R., Nadeem Javaid, N. Amjad, Muhammad Akbar, Zahoor Ali Khan, and U. Qasim. "Impact of acoustic propagation models on depth-based routing techniques in underwater wireless sensor networks." In Advanced Information Networking and Applications Workshops (WAINA), 2014 28th International Conference on, pp. 479-485. IEEE, 2014. 